\title{
Effectiveness of BoNT A in Parkinson's Disease Upper Limb Tremor Management
}

\author{
Fariborz Rahimi, Carina Bee, Derek Debicki, Angela C. Roberts, Priya Bapat, \\ Mandar Jog
}

\begin{abstract}
Objective: One the greatest challenges of BoNT A therapy for tremor lies in the complexity and variation of components involved in tremor movement, and the lack of objective measures to determine these components. This 3 month open-label single injection study aims to couple clinician best judgment with kinematics to improve effect of BoNT A (incobotulinumtoxinA) injection in 7 patients with upper limb Parkinson's disease (PD) tremor. Methods: Injection was guided with clinical and kinematic assessment of tremor using angular wrist position in 3 degrees of freedom: flexion/extension, pronation/supination, and radial/ulnar deviation. Overall tremor severity and change were measured by linear finger acceleration. Results: Kinematic data from static and functional tasks demonstrate no improvement at one month post-injection, but significant improvement at two and three months. Clinical scales across UPDRS Items 20 (1, 2, 3 months post) and 21 (2 months), and spiral drawings ( 3 months) showed significant improvement from baseline, while line drawings did not. Conclusions: This study suggests injection of BoNT A as a viable focal management option for upper limb PD tremor. In addition to clinical judgment, objective quantification of tremor dynamics by kinematics may be a feasible assessment and guidance tool which can be used to optimize injection conditions for focal tremor therapy. Kinematic analysis of tremor across a variety of joints in all degrees of movement may provide important insight into tremor dynamics, allowing optimized, targeted focal therapy.
\end{abstract}

RÉSUMÉ: Efficacité de la BoNT A dans le traitement du tremblement du membre supérieur dans la maladie de Parkinson. Objectif : L'un des plus grands défis dans le traitement du tremblement par la BoNT A est la complexité et la variation des composantes impliquées dans le tremblement et le manque de mesures objectives pour évaluer ces composantes. Le but de cette étude ouverte de 3 mois comportant une seule injection de BoNT A était d'associer le jugement clinique et la cinématique pour améliorer l'effet d'une injection de BoNT A (incobotulinumtoxineA) chez 7 patients présentant un tremblement parkinsonien du membre supérieur. Méthode : L'injection était effectuée à la lumière d'une évaluation clinique et cinématique du tremblement, le poignet étant en position angulaire à 3 degrés de liberté: flexion/extension, pronation/supination et déviation radiale/cubitale. La sévérité globale du tremblement et le changement ont été mesurés par l'accélération linéaire digitale. Résultats : Les données cinématiques des tâches statiques et fonctionnelles n'ont démontré aucune amélioration un mois après l'injection, mais une amélioration significative après deux et trois mois. Les échelles cliniques pour les items 20 (1,2 et 3 mois après l'injection) et 21 (2 mois après l'injection) de la UPDRS et le dessin de la spirale ( 3 mois après l'injection) démontraient une amélioration significative par rapport à l'évaluation faite avant le traitement, alors que le dessin de la ligne n'en montrait pas. Conclusions : Selon les résultats de cette étude, l'injection de BoNT A est une option thérapeutique focale valable pour le traitement du tremblement parkinsonien du membre supérieur. En plus du jugement clinique, une quantification objective de la dynamique du tremblement par la cinématique pourrait être une évaluation réalisable et constituer un outil pratique pour optimiser les conditions d'injection dans le traitement focal du tremblement. L'analyse cinématique du tremblement au niveau de différentes articulations dans tous les degrés de mouvements pourrait fournir des indices importants de la dynamique du tremblement, ce qui permettrait d'offrir un traitement focal ciblé optimisé.

Can J Neurol Sci. 2013; 40: 663-669

Tremor is the most common presenting symptom in Parkinson's disease (PD), and one of the most difficult to treat, affecting the limbs, chin, jaw and tongue ${ }^{1}$. In the upper limbs, tremor-dominant PD patients often present with postural and kinetic tremor simultaneously with rest tremor ${ }^{2,3}$ which may functionally disable task performance, impair sleep, and impede activities of daily living ${ }^{4}$. The conventional treatment of the cardinal symptoms of PD with levodopa and dopamine agonists may substantially improve cardinal symptoms such as akinesia, bradykinesia, rigidity, shuffling of gait, and hypophonia, especially in the early stages of disease; however, PD tremor remains much less responsive ${ }^{5,6}$. Anticholinergic medications (e.g. trihexiphenydyl) can be used in those individuals who have tremor as their predominant symptom. However, these medications have significant side effects including cognitive dysfunction, urinary retention, and xerostomia ${ }^{7,8}$. These symptoms may be intolerable for many, especially elderly

From the Department of Clinical Neurological Sciences (FR, CB, MJ), London Health Sciences Centre; Department of Neurology (DD, MJ), Department of Health Rehabilitation Sciences (ACR), Department of Physiology and Pharmacology (PB), Western University, London, Ontario, Canada.

Received November 19, 2012. Final Revisions Submitted April 23, 2013. Correspondence to: Fariborz Rahimi, London Health Sciences Centre, Department of Clinical Neurological Sciences, 339 Windermere Road, A10-026, London, Ontario, N6A 5A5, Canada. Email: fariborz.rahimi@gmail.com. 
persons with PD, limiting their use in treatment. The need for an effective and well-tolerated treatment of tremor remains a significant unmet need in PD.

Since tremor is often asymmetric and focal, medications used for treatment of focal disorders such as botulinum toxin could be beneficial in the treatment of PD tremor ${ }^{9-12}$. Botulinum toxin type A and B have been well established for the treatment of focal dystonia, with type A demonstrating generally longer duration and better effect ${ }^{13,14}$. While injection of botulinum toxin type A (BoNT A) in the extremities has been studied as a treatment option for tremor ${ }^{15,16}$, it has not been indicated or widely adopted for clinical treatment due to concerns with impairment of limb function from excessive or unwanted muscle weakness despite demonstration of clinical benefit ${ }^{17}$. There are limited studies investigating the use of BoNT A specifically for treating PD tremor. All of these published studies to date have been limited by the number of injection cycles (one or two cycles) and by the duration of follow-up (16 weeks maximum duration) ${ }^{18-20}$.

Tremor is complex, especially in the upper extremity, and its manifestation can change depending on posture, task, and body part $^{21}$. Parkinson disease tremor is often described as "pillrolling"; however, this tremor is not isolated to the fingers. Wrist tremor is more complicated as the wrist joint can flex-extend, rotate and deviate side to side, simultaneously. Tremor of the elbow and more proximal and axial aspects of tremor become increasingly harder to elucidate due to the biomechanics of the joints involved. Hence, proper characterization of the tremor based on visual inspection alone is a daunting task for the clinician.

In considering BoNT A as a treatment for tremor in PD, appropriate muscle selection, dosing per injection site, and localization of muscles for injection are dependent on correct movement characterization ${ }^{22}$. In order to achieve this, the joint (e.g. elbow), the direction of the predominant movement (e.g. extension), and the relative contributions of combinations of muscles to the overall tremor must be characterized ${ }^{21}$. Currently, characterization is done entirely on the basis of visual inspection and "gestalt" of the injector. Due to the numerous factors involved, such as the variability of the tremor and differences across patients, visually-guided injections are prone to failure and side effects, limiting the use of BoNT A for tremor treatment ${ }^{13,23}$

Kinematic methodology is well established for studying the dynamics of movement in the upper limb ${ }^{24,25}$. Technological advances have made this a viable option in the objective characterization of complex movements such as tremor. Using both clinical experience and data provided by kinematic decomposition of tremor, we have conducted a longitudinal single-injection open label preliminary study of the use of BoNT A for the treatment of PD tremor in the upper extremity. We hypothesized that additional information provided to the clinician in the form of kinematic data along with injector's clinical assessments will improve characterization of PD tremor, and produce better outcomes than prior studies.

\section{METHODS}

This current preliminary study uses an open label single injection paradigm with monthly follow-up over three months.
The total study duration and tremor follow-up was three months with a total of four study visits. All participants with PD were scheduled for their data collection visits in their medication "ON" state. At each study visit, participants completed clinical and kinematic tremor assessments. Participants received BoNT A injections during their first (baseline) visit. All patients were injected in their tremor dominant limb with incobotulinumtoxin A, mixed in the dose of $1: 1$ dilution (1cc of saline per 100 units of BoNT A). All patients were injected by a single injector (MJ) under electromyographic (EMG) guidance (1" long 30g injectable EMG needle using a Clavis ${ }^{\circledR}$ portable EMG machine). Subsequent study visits were scheduled at four week intervals up to three months post-injection (1M, 2M, 3M).

\section{Participants}

A convenience sample of ten patients with PD were recruited from the Movement Disorders Centre at the London Health Sciences Centre, Canada. The study protocol was approved by the Western University Health Sciences Research Ethics Board (REB\#16884). Inclusion criteria included participants: on stable medication management for a minimum of six months prior to enrolment, with none withheld or adjusted at the time of the study, having tremor as their primary and most bothersome symptom, and BoNT A naïve. Exclusion criteria were applied for those with: prominent and predominant finger tremor, previous deep brain stimulation (DBS), and existing pharmacological therapy with tremor-inducing side effects (eg. lithium, valproate, etc.).

\section{Clinical Scale Assessment}

Clinical scales employed in this study were as follows. The Tremor Assessment Form Rating Scale (TAF) included straight line and Archimedes spiral drawings. Tremor scores were evaluated by a separate assessor for all patients as per the $\mathrm{TAF}^{26}$. Additional subjective reports of upper limb weakness were recorded at all visits. Unified Parkinson Disease Rating Scale (UPDRS) was administered by a movement disorders specialist (MJ) for Items 20 (hand only) and 21. Researchers scoring the scales were blinded to results of scales from other data collection points in the study.

\section{Kinematic Assessment}

Participants also performed a series of tasks conducted in the seated position used to elucidate tremor in the upper limb position including postures used as part of a routine neurological examination. Tasks were designed to observe rest tremor, postural tremor, kinetic tremor and tremor under a loaded (with a weighted load) condition (Figure 1B-G). Rest and postural tasks were measured as static tasks (Figure 1B-E), whereas kinetic and loaded tasks were measured as functional tasks (Figure 1F-H). Although the functional tasks are not equivalent to all activities of daily living, these tasks were intentional and goal-oriented and hence termed functional.

A series of motion recording sensors were placed as shown in Figure 1A. Tremor was assessed by measuring angular position about the wrist, and by measuring linear acceleration at the distal phalanx. An electrogoniometer at the wrist joint measured wrist flexion/extension (F/E) and radial/ulnar deviation (R/U) (SG65, 


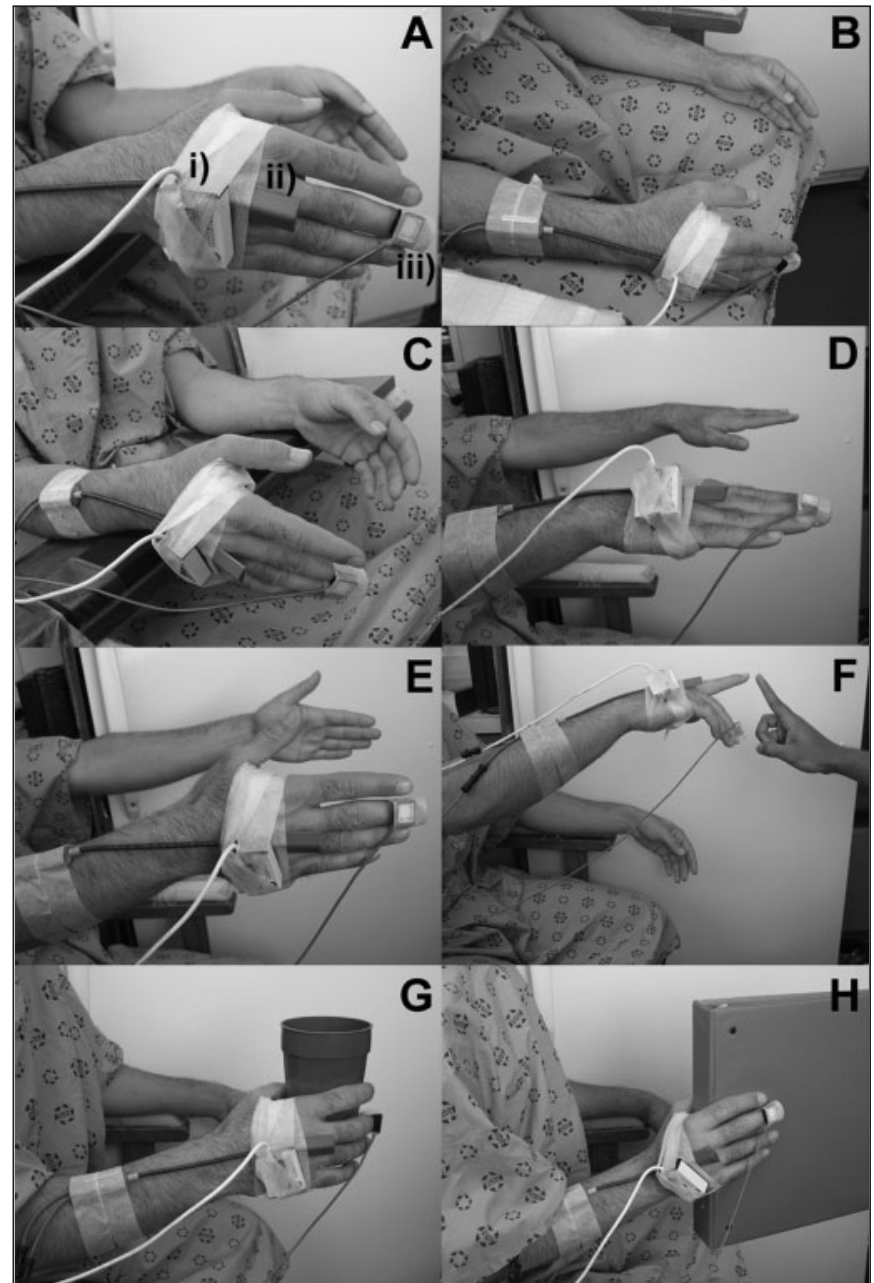

Figure 1: A) Experimental setup and sensor placement: i) inclinometer, ii) electrogoniometer, iii) accelerometer; $B$ ) relaxing the forearm on the lap in neutral position (Rest-1); C) relaxing the forearm at armrestheight in neutral position (Rest-2); D) shoulders flexed at $90^{\circ}$ with arms extended anteriorly and pronated (Posture-1); E) shoulders flexed at $90^{\circ}$ with arms extended anteriorly in neutral position (Posture-2); F) alternating between touching researcher's moving finger and participant's own nose (Kinetic); G) holding an empty plastic cup (33g) (Load-1); H) holding a binder by the spine (800g) (Load-2)

Biometrics Ltd), while an inclinometer attached to the dorsal surface of the hand measured forearm pronation/supination $(\mathrm{P} / \mathrm{S})$ (Noraxon $\left.{ }^{\circledR}\right)$, providing the third angular degree of freedom of motion about the wrist. While the electrogoniometer recorded relative motion of the wrist and the forearm, the inclinometer utilized a global inertial frame of reference. A lightweight 3D accelerometer collected distal finger movements $(3 \mathrm{D}, 6 \mathrm{~g}$, Noraxon $($ ). All data were sampled at $1500 \mathrm{~Hz}$, and acquired by TeleMyo $^{\mathrm{TM}}$ 2400T G2 and PC interface (MyoResearch XP Master Edition 1.08.09, Noraxon $\left.{ }^{\circledR}\right)$. All sensors were attached using medical grade tape. Calibration of the sensors for two degrees of freedom (DOF) was done with the hand placed against a fixed vertical plane in neutral F/E, R/U and P/S for five seconds duration. The first five tasks were repeated in series consecutively for a total of three times (trials), for ten seconds duration for each task trial (Figure 1B-F). These were then followed by a single trial of Load-1 (33g) and a single trial of Load-2 (800g) for ten seconds each (Figure 1G).

\section{Kinematic Data Analysis}

Signal processing was performed in MatLab ${ }^{\circledR}$ (MathWorks, R2011a). For each participant data file, the segments corresponding to each trial were extracted for every task. Each segment included three angular position signals for the wrist and three linear acceleration signals for the finger. For each angular position signal, the mean value during neutral position calibration was subtracted before further processing. Both angular position and acceleration signals were band-pass filtered $(2-20 \mathrm{~Hz})$. The three dimensions of angular position at the wrist and linear acceleration at the finger were combined (RMS) to provide overall wrist angular tremor and overall tremor severity at the finger, respectively. All measures of tremor at the wrist and at the distal phalanx were used for characterization of the tremor in order to guide the injection.

\section{Injection Determination}

For the baseline visit, kinematic analysis data were plotted graphically and presented to the injector post kinematic and clinical assessment, but prior to injection (Figure 2). Classical tasks of Rest- 1 and Posture-1 were chosen a priori to be representative of tremor movement for each participant (Figure 2). Percent contribution for each of the three components to wrist tremor was determined with respect to the summed 3D angular amplitude $(\mathrm{F} / \mathrm{E}+\mathrm{R} / \mathrm{U}+\mathrm{P} / \mathrm{S})$ (Figure 2).

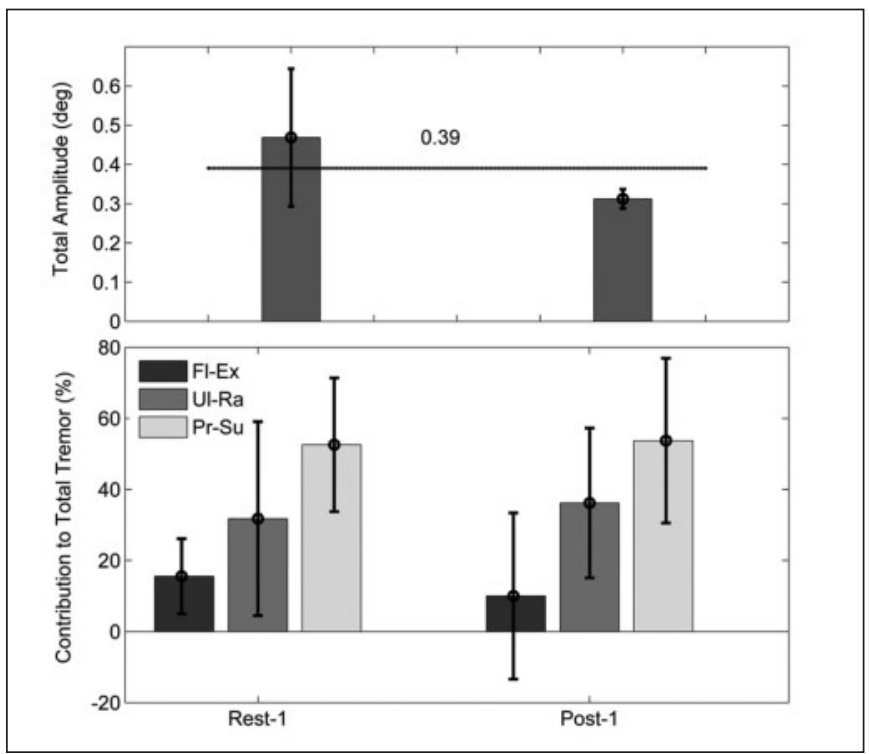

Figure 2: Graphical representation of kinematic data as shown to injector. Patient-5, tremor in Rest-1 and Posture-1 at baseline visit as deconstructed into $3 \mathrm{DOF}(F / E, P / S$, and $R / U)$ : A) The combined $R M S$ amplitude of the three DOF over all the three trials at rest and posture are presented. The horizontal line is the average of the two bar graphs (3 trials of rest and posture each). B) Percent contribution of each of the 3 DOF is shown for the overall tremor. 
Clinical scale assessment data and kinematic data were presented to an experienced movement disorders neurologist trained in BoNT A injections (MJ) for each patient at baseline for injection in their tremor-dominant forearm. The same injector then performed the UPDRS.

The kinematic data gave the injector a clearer understanding of the contributions of the joints involved in the tremor and the bias towards flexion, extension etc. Kinematics and the UPDRS together gave the impression for severity. The other clinical scales provided a functional measure to the injector as to which movements affected the patient most. Knowing anatomically which muscles may be responsible for the kinematic and clinical parameters mentioned, together, the injector then planned the muscles to be injected. Finally, the actual dose per muscle was determined based on injector experience.

\section{Statistical Analyses}

While tremor severity was measured as both wrist angular amplitude as well as finger acceleration, the latter was chosen as the best measure of overall tremor severity given it optimally captures all motion as it records from the most distal limb location. Pearson's correlation was used to validate finger acceleration to be as effective as wrist angle in representing overall tremor.

Load-1 and Load-2 data were single trials. All other tasks were averaged across the three trials per patient per visit. Both kinematic and clinical data were imputed for 3 unavailable data points by multiple data imputation method (STATISTICA 8.0®).

For finger acceleration kinematic data, change values at each timepoint referenced to baseline were entered into statistical analysis.

\section{Percentage improvement $(\%)=\left(x_{0}-x_{1}\right) / x_{1} \times 100 \%{ }^{1}$}

Data were grouped into two subgroups based on task (Figure 1), all static tasks (Rest-1, Rest-2, Posture-1 and Posture-2) and all functional tasks (Kinesis, Load-1 and Load-2), at one month, two month, and three months post-injection. Percent improvement in each group of tasks and each timepoint was compared against zero (no improvement) using two-tailed independent t-tests $(=.05)$. Descriptive and test statistics, including Cohen's d for effect size, are presented along with boxplots as visual representation of the spread of data and outliers per group of tasks per timepoint. For each clinical scale, a repeated measures analysis of variance (ANOVA) was performed across all timepoints. Partial eta-squared was reported for effect sizes. Post-hoc analysis was conducted by Tukey's HSD, with significance level $\alpha=.05$.

\section{ReSUlts}

\section{Demographics}

A total of ten participants were screened for the study and seven patients with PD were recruited (five male, six righthanded, mean age $=59 \pm 7.7$ years). Demographics are summarized in Table 1.

All patients were injected in the tremor dominant upper limb. The mean dose of BoNT A was 97 $\pm 52 \mathrm{U}$ (Table 2). Muscles injected are shown in Table 2. The predominant muscles injected were ECU and ECR.

\section{Side Effects}

Five of seven participants subjectively reported a side effect of finger weakness. Only one of the five participants felt the weakness to be significant indicating a disruption to the activities of daily living while the other four did not feel the weakness to be disruptive (Table 2). The remaining two participants did not report any subjective weakness. Median timepoint of when the patients reported weakness was at one month post-injection. Two cases of weakness remained at two months and no participants reported weakness at three months. No other unwanted effects associated with the injections were observed.

\section{Kinematic Results}

Angular amplitude at the wrist and linear acceleration at the third finger were correlated by Pearson's coefficient across all four visits $(r=0.89 \pm 0.01)$. Therefore, only change in finger acceleration is represented as the objective measure of BoNT A effect.

Table 1: Participant demographics and clinical scale scores pre and post injection

\begin{tabular}{ccccccccccc}
\hline $\begin{array}{c}\text { Subject } \\
\text { ID\# }\end{array}$ & Age & Gender & $\begin{array}{c}\text { Yrs with } \\
\text { Tremor }\end{array}$ & $\begin{array}{c}\text { Assessed } \\
\text { Side }\end{array}$ & \multicolumn{3}{c}{ Baseline-Score } & \multicolumn{4}{c}{ Optimum Post-Score (M) } \\
\hline 1 & 47 & M & 11 & R & 6.5 & 3 & 2 & $3.5(2)$ & $1(3)$ & $1(1,3)$ \\
2 & 66 & M & 3 & R & 5.0 & 0 & 0 & $3(2)$ & 0 & 0 \\
3 & 55 & M & 1 & R & 3.0 & 0 & 0 & $1.5(2)$ & 0 & 0 \\
4 & 57 & F & 6 & L & 5.5 & 4 & 4 & $4(1)$ & 4 & 4 \\
5 & 71 & M & 5 & R & 3.0 & 1 & 0 & $0(1)$ & $0(1,3)$ & 0 \\
6 & 58 & M & 7 & R & 6.5 & 1 & 0 & $2(3)$ & $0(2,3)$ & 0 \\
7 & 60 & F & 6 & R & 4.0 & 1 & 1 & $1(1)$ & $0(3)$ & $0(2)$ \\
\hline Mean \pm SD & $59 \pm 7.7$ & $5 \mathrm{M}$ & $5.6 \pm 3.1$ & $6 \mathrm{R}$ & $4.8 \pm 1.5$ & $1.4 \pm 1.5$ & $1.0 \pm 1.5$ & $2.1 \pm 1.4$ & $0.7 \pm 1.5$ & $0.7 \pm 1.5$ \\
\hline
\end{tabular}

Imputed data were not considered for optimum post-scores. (M) represents the month after injection that showed the best benefit for every patient. 
Table 2: Injection dosage, muscle selection, optimum improvement, and weakness as determined by injector across all participants

\begin{tabular}{cccccccccccccc}
\hline $\begin{array}{c}\text { Subject } \\
\text { ID\# }\end{array}$ & FCR & FCU & ECR & ECU & PRT & SUP & BIC & TRI & FDS & FPL & Static & Functional & Weakness \\
(M)
\end{tabular}

* = Patient report of significant finger weakness. Only one patient had significant weakness while the others did not. All the dosages are in units of Xeomin. Individual optimum improvement was averaged for each task group

Kinematic data from static tasks and functional tasks demonstrate no significant improvement at one month postinjection. However, significant improvement was observed at two months and three months post-injection. The data for percent improvement in tremor amplitude (acceleration) for the two groups of tasks for one, two, and three months post-injection are presented in Figure 3. Only 9 out of 106 data points (outliers) were not used for analysis as they were more than two standard deviations above and below the mean improvement. Both the data and outliers are shown in the boxplot. Individual best percent improvements are presented in Table 2. The median time for best improvement for static tasks was at month 2 and for

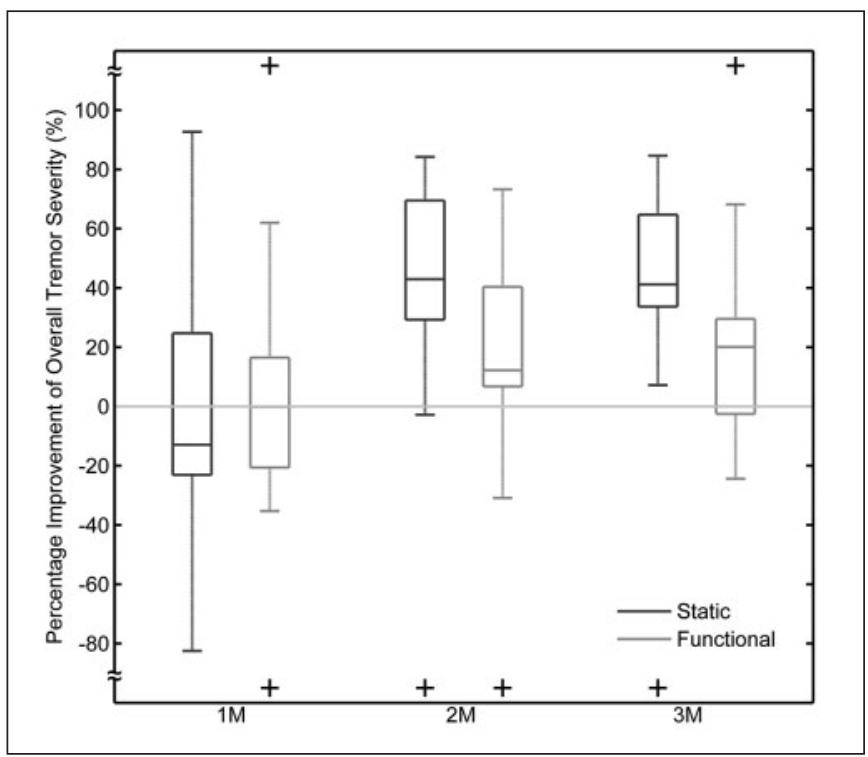

Figure 3: Percentage improvement of overall tremor severity as an effect of BoNT A for all static and functional tasks at 1 month, 2 month, and 3 month time points post-injection with respect to baseline $(0 \%)$. These have been grouped per task per month. Outliers have been removed from the boxplots but are represented as a "+" as both good and bad outliers. Number of outlier data points: $9 / 106=8 \%$ of all data. functional tasks at month 2 . The statistics are as follows: one month (Static: $M=-1, S D=47,95 \% C I[-20.6,18.8], t(21)=-$ $.09, p=.93, d=.02$; Functional: $M=-1, S D=25, C I[-12.6$, 11.1], $t(16)=-.12, p=.90, d=.03$ ); two months (Static: $M=45$, $S D=25, C I[32.9,56.4], t(17)=7.45, p<.001, d=1.80$; Functional: $M=21, S D=29, C I[6.2,35.4], t(14)=2.79, p=$ $0.01, d=.75$ ); three months (Static: $M=46, S D=24, C I[32.9$, 59.5], $t(12)=6.81, p<.001, d=1.97$; Functional: $M=16, S D=$ $25, C I[1.4,29.9], t(11)=2.15, p=0.05, d=.65)$.

\section{Clinical Results}

Significant change was observed for three of four measures: UPDRS Item $20\left(\mathrm{~F}(3,18)=7.79, p=.001, \eta^{2}=.56\right)$, Item 21 $\left(\mathrm{F}(3,18)=3.98, p=.024, \eta^{2}=.40\right)$, and spiral drawing $(\mathrm{F}(3,18)$ $=3.71, p=.031, \eta^{2}=.38$ ) (Figure 4). Changes in line drawing were not significant $(\mathrm{F}(3,18)=1.48, p=.254)$. Post-hoc analysis showed significant improvement across timepoints, represented

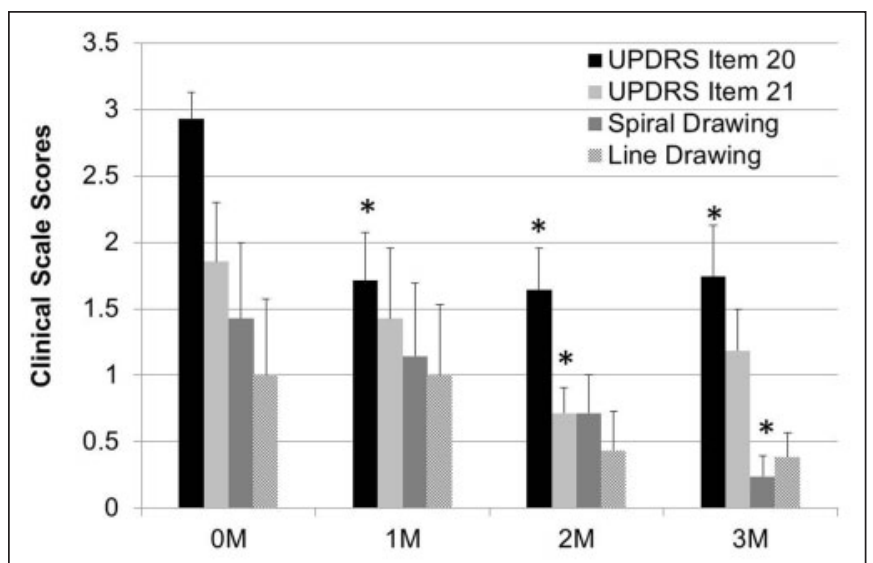

Figure 4: Effect of BoNT A on tremor severity in the injected hand across all participants as measured by clinical scales: UPDRS Items 20 (hand) and 21, and straight line and Archimedes spiral drawing components of the Tremor Assessment Form. Asterisks represent post-hoc significance within measures. 
by a reduction in the clinical scale score, in UPDRS Item 20 at one month, two, three, with respect to baseline ( $p=.005, p=$ $.003, p=.007$, respectively $)$, Item 21 at three month $(p=.016)$, and spiral drawing at four month with respect to baseline ( $p=$ .028) (Figure 4). A trend across scales showed prolonged improvement effect for three months post-injection (Figure 4). Optimum improvement was observed (based on median scores) at two months post-injection.

\section{DISCUSSION}

This is the first study to report the effect of incobotulinumtoxinA in the treatment of PD tremor. In this study, participants were injected once and followed for three months post-injection to monitor wearing-off of BoNT A effect. Finger weakness was also reported to monitor side effects, and was found isolated to the injected hand and forearm only. Both kinematic and clinical endpoints demonstrate significance across time points. However, in contrast to previously published studies, significant improvement from baseline kinematic and clinical data was only observed at two months and three months post-injection, whereas most studies have reported maximum effect at four to six weeks, and lasting up to three months post-injection ${ }^{10}$. In addition, the results from three months post-injection suggest that clinical and kinematic scales do not fully return to baseline, suggesting effect of BoNT A to surpass three months (Figure 4). Wearing-off of BoNT A may be inferred from slight worsening in UPDRS Item 20 at three month post-injection, but this trend is not consistent across measures (Figure 4). A longer duration study would be necessary to examine the extended effect and complete wearing-off of BoNT A beyond three months.

Finger acceleration is the most commonly used kinematic measure of tremor ${ }^{27}$. Hence finger acceleration has been used in this study as the primary outcome measure. However, the measurement of finger acceleration is not capable of allowing tremor deconstruction as demonstrated in this paper. Therefore, wrist kinematics recording is essential. Angular data from the wrist has been used in this study as a means of deconstructing tremor dynamics into direction components in order to determine injection pattern.

Our data show a strong correlation between angular wrist amplitude and finger acceleration. This validates the use of finger acceleration as a robust measure of overall tremor severity. This is an important finding implying that injection of tremor in the wrist muscles and the reduction in angular amplitude at the wrist as recorded by the kinematics is well reflected in the reduction of finger tremor amplitude. This methodology is being utilized in the next phase as a larger multiinjection longitudinal study that further seeks to optimize injection parameters (muscle groups and dosage).

Kinematic data from static tasks and functional tasks demonstrate significant improvement of PD tremor with BoNT $\mathrm{A}$ at two and three months post-injection. This suggests that botulinum therapy not only improves tremor upon classical tasks such as Rest- 1 and Posture-1 up to three months, but extends its improvement to functional tasks. Although not specifically examining all the tasks involved in activities of daily living, holding a glass, which is a load, may represent similitude to what affects patients in task performance with tremor.
Despite the fact that participants were assessed around the same time of day and in a consistent manner, fluctuating tremor with mood, stress level, etc. can be expected to contribute to variability in kinematic measures. Such variability can be observed in both positive and negative outliers in percent improvement (Figure 3). Presence of higher tremor amplitude post injection (Rest-1 and Rest-2, specifically for this participant) might demonstrate excessive and disproportionate worsening, simply based upon variability in the patient's tremor. This may also account for disproportionate improvement. Due to such variability of data, such outliers were removed.

Clinical findings also support these objective results. ANOVA determined improvement to be significant across UPDRS Items 20 and 21, and spiral drawings of clinical scales. A larger sample size is necessary to generate more power to further validate these findings. The UPDRS was scored by the injector in a blinded fashion to previous visit scores. Complete blinding of the injector as well as of the researcher to all clinical scales of prior visits, or different injecting and evaluating physicians, may minimize any experimenter bias. However, this bias can be removed with objective, kinematically based assessments.

Four studies have reported the use of onabotulinumtoxinA for the treatment of PD hand tremor with relatively small sample sizes of $3,1,12$ and $12^{9,18,19,28}$. These studies have used clinical scales, and of these, two have included accelerometry as well ${ }^{9,19}$. Henderson et al showed an average of $22 \%$ reduction in clinical tremor score, for rest and posture, four weeks post-injection. For the three participants, the same scores maintained an average of $17 \%$ reduction after ten weeks. In another study of 12 PD patients, Trosch and Pullman reported 13\% average decrease in accelerometric tremor amplitude over rest and posture trials (with a small-to-medium effect size, $\mathrm{d}=.44$ ). They did not find significant changes in any of the clinical scales after injection ${ }^{9,18,19,28}$. In our study, highest average improvement in static tasks, which included postural tremor, was $46 \%$ at three months post-injection (with a very large effect size of $d=1.97$ ) and all other measures showed moderate to large effect sizes (see results). Previous studies also report postural tremor improvement by finger accelerometry (11\%, 12 PD) and functional improvement by self-report (17\%, 12 PD $)^{9,19}$. However, these studies showed minor or no significant change in clinical rating scales, respectively. Our study, across UPDRS items 20,21, and spiral drawing (clinical scales) demonstrated small to medium effect sizes for improvement at months 2 and 3 . The limitations of our study are the small sample size, single non-placebo controlled and non-blinded injections and no treatment comparator. However, the sample size is similar to other reported studies in the literature as discussed above. In addition, we used objective kinematic measures not only to provide some guidance for selection of the injections but also for all of the follow-up potentially reducing subjective bias by using clinical ratings alone. It is also possible that the tremor itself changed at visits and this variability was the reason for the apparent reduction. However, the effect sizes across all measures were robust.

At present, literature on PD tremor injection with BoNT A has not shown similar success as with essential tremor. Supporting evidence from previous tremor analysis data suggest 
that PD tremor is multi-component, especially compared with $\mathrm{ET}^{21}$. However, most studies of focal hand tremor emphasize only the injection of flexor and extensor muscle groups. Jankovic and Sheffield (2009) have suggested that injection of biceps brachii be considered to address the supination component in PD tremor ${ }^{19}$. In our study, we have targeted pronator and supinator muscle groups, addressing tremor complexity in all degrees of freedom.

It is recognized that access to kinematic analysis technology and expertise is limited, even within many dedicated movement disorders clinics. Nevertheless, the current study highlights the addition of pronators and supinators along with even the larger muscles of the arm such as biceps and triceps may need to be considered for injection in patients with PD tremor. It is possible that keeping the doses of forearm injection low while adding injections into these larger muscles may be beneficial. For this study, we have specifically omitted participants with prominent and predominant finger tremor. Such injections would inherently produce weakness in the fingers and would not be desirable for functional improvement in patients. This is a limitation of BoNT A injections for tremor, if the tremor is predominantly in or limited to the fingers.

The results from the current study have prompted the development of a longitudinal study to further examine the use of BoNT for treatment of PD tremor. We suggest that early intervention with focal BoNT A injection, in our case BoNT A, in patients where PD tremor is the dominant symptom, is an important feasible treatment option which has not yet been utilized to its full advantage. Objective kinematic analysis appears to be an advantageous adjunct to the traditional clinical assessment, which allows the clinician to more accurately target specific muscle groups for injection. Future studies using this technology will further identify which muscles groups most commonly contribute to PD tremor.

\section{ACKNOWLEDGMENTS/DisClOSURE}

The present study was partially funded by a research grant from Merz Pharmaceuticals. BoNT A (Xeomin) was provided in kind by Merz Pharmaceuticals. The authors would also like to acknowledge the contribution of the patients and staff at the National Parkinson Foundation Centre of Excellence, London Health Sciences Centre, London, Ontario, Canada.

Dr. Jog receives speaker honoraria from Merz Pharmaceuticals, Allergan, Novartis, Teva Neurosciences and Abbott. Dr. Rahimi has received travel reimbursements and speaker fees from Merz Pharmaceuticals and Teva Neurosciences.

\section{REFERENCES}

1. Jankovic J. Parkinson's disease: clinical features and diagnosis. J Neurol Neurosurg Psychiatry. 2008;79(4):368-76.

2. Milanov I. A cross-over clinical and electromyographic assessment of treatment for parkinsonian tremor. Parkinsonism Relat Disord. 2001;8(1):67-73

3. Kraus PH, Lemke MR, Reichmann H. Kinetic tremor in Parkinson's disease--an underrated symptom. J Neural Transm. 2006;113(7): 845-53.

4. Fleischman DA, Wilson RS, Schneider JA, Bienias JL, Bennett DA. Parkinsonian signs and functional disability in old age. Exp Aging Res. 2007;33(1):59-76.

5. Politis M, Wu K, Molloy S, G. Bain P, Chaudhuri KR, Piccini P. Parkinson's disease symptoms: the patient's perspective. Mov Disord. 2010;25(11):1646-51.
6. Miyasaki JM, Martin W, Suchowersky O, Weiner WJ, Lang AE. Practice parameter: initiation of treatment for Parkinson's disease: an evidence-based review: report of the Quality Standards Subcommittee of the American Academy of Neurology. Neurology. 2002;58(1):11-7.

7. Katzenschlager R, Sampaio C, Costa J, Lees A. Anticholinergics for symptomatic management of Parkinson's disease. Cochrane Database Syst Rev. 2003;(2):CD003735.

8. Schadt CR, Duffis EI, Charles PD. Pharmacological treatment of disabling tremor. Expert Opin Pharmacother. 2005;6(3):419-28.

9. Henderson JM, Ghika JA, Van Melle G, Haller E, Einstein R. Botulinum toxin A in non-dystonic tremors. Eur Neurol. 1996;36 (1):29-35.

10. Sheffield JK, Jankovic J. Botulinum toxin in the treatment of tremors, dystonias, sialorrhea and other symptoms associated with Parkinson's disease. Expert Rev Neurother. 2007;7(6): 637-47.

11. Jankovic J. Disease-oriented approach to botulinum toxin use. Toxicon. 2009;54(5):614-23.

12. Keir J, Giele HP. Role of botulinum toxin in hand and upper limb disorders relevant to hand therapy. Hand Therapy. 2009;14(2): 32-8.

13. Das CP, Truong D, Hallett M. Treatment of focal hand dystonia. In: Truong D, Dressler D, Hallett M, editors. Manual of Botulinum Toxin Therapy: Cambridge University Press; 2009.

14. Patel S, Martino D. Cervical dystonia: From pathophysiology to pharmacotherapy. Behav Neurol. 2012.

15. Simpson DM, Blitzer A, Brashear A, et al. Assessment: Botulinum neurotoxin for the treatment of movement disorders (an evidence-based review): report of the Therapeutics and Technology Assessment Subcommittee of the American Academy of Neurology. Neurology. 2008;70(19):1699-706.

16. Van Der Walt A, Sung S, Spelman T, et al. A double-blind, randomized, controlled study of botulinum toxin type A in MSrelated tremor. Neurology. 2012;79(1):92-9.

17. Jankovic J, Schwartz K, Clemence W, Aswad A, Mordaunt J. A randomized, double-blind, placebo-controlled study to evaluate botulinum toxin type A in essential hand tremor. Mov Disord. 1996;11(3):250-6.

18. Jankovic J, Schwartz K. Botulinum toxin treatment of tremors. Neurology. 1991;41(8):1185-8

19. Trosch RM, Pullman SL. Botulinum toxin A injections for the treatment of hand tremors. Mov Disord. 1994;9(6):601-9.

20. Brin MF, Lyons KE, Doucette J, et al. A randomized, double masked, controlled trial of botulinum toxin type A in essential hand tremor. Neurology. 2001;56(11):1523-8.

21. Rahimi F, Debicki D, South A, Bee C, Bapat P, Jog MS. Dynamic decomposition of motion in essential and parkinsonian tremor. Transactions on Neural Systems \& Rehabilitation Engineering. 2012.

22. Rozman J, Bartolic A, Ribaric S. A new method for selective measurement of joint movement in hand tremor in Parkinson's disease patients. J Med Eng Technol. 2007;31(4):305-11.

23. Fishman PS. Paradoxical aspects of parkinsonian tremor. Mov Disord. 2008;23(2):168-73.

24. Supuk T, Bajd T, Kurillo G. Assessment of reach-to-grasp trajectories toward stationary objects. Clin Biomech (Bristol, Avon). 2011;26(8):811-8.

25. Casellato C, Zorzi G, Pedrocchi A, Ferrigno G, Nardocci N. Reaching and writing movements: sensitive and reliable tools to measure genetic dystonia in children. J Child Neurol. 2011;26 (7):822-9.

26. Society MD. 1 May 2009 [cited; Available from: http://www.mdvu. org/library/ratingscales/et/et_taf.pdf

27. Wong WY, Wong MS, Lo KH. Clinical applications of sensors for human posture and movement analysis: a review. Prosthet Orthot Int. 2007;31(1):62-75.

28. Pullman SL, Greene P, Fahn S, Pedersen SF. Approach to the treatment of limb disorders with botulinum toxin A. Experience with 187 patients. Arch Neurol. 1996;53(7):617-24. 